Unfallchirurg 2017 · 120:561-572

DOI 10.1007/s00113-017-0372-8

Online publiziert: 22. Juni 2017

(c) Der/die Autor(en) 2017. Dieser Artikel ist eine Open-Access-Publikation.

\section{Redaktion}

A. Trampuz, Berlin

C. Willy, Berlin

CrossMark

\section{Hintergrund}

Die Entwicklung antibiotikahaltiger Werkstoffe in der Knochen- und Gelenkchirurgie geht auf Buchholz und Engelbrecht [7] zurück, die Ende der 60er Jahre des letzten Jahrhunderts erstmals Versuche mit Zumischungen von Antibiotika in Polymethylmethacrylat(PMMA)-Knochenzement durchführten und eine drastische Senkung der Anzahl periprothetischer Infektionen in der Endoprothetik beobachteten. Auch in der Unfallchirurgie wurde eine signifikante Reduktion der Inzidenz akuter Knocheninfektionen und Osteomyelitisfälle bei offenen Brüchen mit erheblicher Schädigung des Weichteilgewebes (Gustilo-Typ-IIIB- und -IIICFrakturen) beschrieben, wenn zusätzlich zum chirurgischen Débridement und zur systemischen Antibiotikaprophylaxe antibiotikabeladene Knochenzementketten vor Ort eingesetzt wurden [34, $35,53,58]$.

\section{) Mit antibiotikahaltigem Knochenzement ist eine Infektionsratenreduktion um ca. $50 \%$ möglich}

Die zurzeit verlässlichsten und aktuellsten Daten bieten die skandinavischen Endoprothesenregister. Sie belegen den Nutzen antibiotikahaltiger Knochenzemente im Rahmen der primären Endoprothetik zur Prophylaxe periprothetischer Infektionen [16, 31]. Eine Metaanalyse aus den USA zeigte, dass durch die Anwendung antibiotikahaltiger Kno-

\author{
Klaus-Dieter Kühn ${ }^{1}$. Nora Renz ${ }^{2}$. Andrej Trampuz ${ }^{2}$ \\ ${ }^{1}$ Universitätsklinik für Orthopädie und Orthopädische Chirurgie, Medizinische Universität Graz, Graz, \\ Österreich \\ ${ }^{2}$ Centrum für Muskuloskeletale Chirurgie, Charité - Universitätsmedizin Berlin, Campus Virchow-Klinikum, \\ Berlin, Deutschland
}

\title{
Lokale Antibiotikatherapie
}

chenzemente eine Reduktion der Infektionsraten in der primären Hüftendoprothetik um ca. $50 \%$ möglich ist [55]. Antibiotikabeladene Knochenzemente gehören seit vielen Jahren zum chirurgischen Standard der Implantatfixierung. In der Regel kommen dabei breit und konzentrationsabhängig wirkende Aminoglykoside (zumeist Gentamicin oder Tobramycin) zum Einsatz.

Für septische Revisionseingriffe sind festgelegte Therapiealgorithmen definiert $[56,60]$. Bei chronischen Infektionen werden neben der radikalen chirurgischen Entfernung des infizierten und nekrotischen Gewebes in der Regel adjuvant systemische und lokale Antibiotika eingesetzt, um die Keimzahl im betroffenen Gelenk weiter zu reduzieren. Im einseitigen Wechsel ermöglichen antiinfektiv beladene Knochenzemente die Verankerung der Revisionsprothese, ohne dass das Implantat und der Zement mikrobiell wiederbesiedelt werden. Im mehrzeitigen Verfahren werden Knochenzementspacer als temporäre Platzhalter in der Interimsphase zwischen Ausbau der infizierten und Einbau der Revisionsprothese verwendet. Auch hier werden die Zemente während der intraoperativen Herstellung der Spacer keimgerecht und auf der Basis einer antibiotischen Resistenztestung zusätzlich mit Antiinfektiva kombiniert. Ziel ist auch hier, eine hohe lokale antimikrobielle Wirkstoffkonzentration über die gesamte Zeit des "Totraummanagements“ $\mathrm{zu}$ erzielen, um die Spaceroberfläche vor einer erneuten Keimbesiedelung zu schützen und im umliegenden Gewebe noch vorhandene Keime in ihrer Anzahl weiter deutlich zu reduzieren.

\section{Überblick über lokale Wirkstoffträger}

Auch wenn für Knochenzemente auf PMMA-Basis die meisten Erfahrungswerte vorliegen, werden in der septischen Knochenchirurgie auch lokale Wirkstoffträger aus anderen Werkstoffen perioperativ eingesetzt.

\section{Autologes oder allogenes Knochentransplantat}

Autologes oder allogenes Knochentransplantat (nativ, lyophilisiert, thermodesinfiziert) nimmt je nach Oberfläche flüssige Stoffe auf und gibt diese verzögert ab [11]. Antibiotisch beladene Knochenchips wurden erstmals lokal zur Behandlung einer Osteomyelitis eingesetzt und dabei mit Penicillin kombiniert [14, 54, 63]. Inzwischen gibt es zahlreiche Berichte über eine erfolgreiche Infektsanierung und Auffüllung von Knochendefekten im Zuge einer Osteomyelitistherapie durch Verwendung von allogenem spongiösen Knochenmaterial, das wahlweise mit verschiedenen Antibiotika imprägniert wurde [70]. Hydrophobe (fetthaltige) Stoffe mit adhäsiven Charakter können an der Oberfläche von Knochenchips anhaften und entsprechend der Löslichkeit der Fette Antibiotika freisetzen. Dabei können auch Wirkstoffbestandteile in den Knochen eindringen und zusätzlich einen Retardeffekt bewirken [9]. Grundsätzlich können hydrophile und hydrophobe Wirkstoffe mit Knochenchips kombiniert werden. Der leicht lösliche Wirkstoffanteil wird dabei unmittelbar nach Implantation freigesetzt, während die schlechter lösliche Komponente für eine verzögerte 
Elution über mehrere Tage sorgt [12]. Aus diesen Gründen ist eine Imprägnierung autologer oder allogener Knochenchips mit Antibiotika sinnvoll, valide klinische Studien dazu fehlen jedoch.

\section{Streuung von Antibiotika in die operative Wunde}

In mehreren Studien konnte gezeigt werden, dass Streuung von Antibiotika in Puderform in die operative Wunde zu einer signifikanten Reduktion von postoperativen Infektionen führt, insbesondere in der Wirbelsäulenchirurgie [33, 45, 49, 57]. Durch lokale Gabe schlecht resorbierbarer Antibiotika (z. B. Vancomycin, Gentamicin) werden für einige Stunden bis Tage hohe Antibiotikakonzentrationen im Operationsgebiet erreicht, ähnlich wie bei einer intramuskulären Injektion. Die Gefahr einer toxischen Wirkung auf Osteoblasten bei hoher lokalen Konzentrationen kann jedoch nicht ausgeschlossen werden [18].

\section{Knochenersatzwerkstoffe}

Knochenersatzwerkstoffe (KEW) kommen dann in Betracht, wenn autologes bzw. allogenes Material zur Auffüllung von Knochendefekten nicht oder in nicht ausreichenden Mengen zur Verfügung steht $[4,63]$. Auch eine Mischung beider Materialien ist möglich und kann durchaus sinnvoll sein. Aus mikrobiologischer Sicht ist die Dosierung von Wirkstoffen in KEW reproduzierbar einstellbar. Der Anwender kann sich auf einen festgelegten deklarierten Wirkstoffgehalt stützen. Dies gilt auch, wenn KEW, die Wirkstoffe enthalten, mit körpereigenem Knochen kombiniert werden.

Kalziumverbindungen als Grundstoff vieler KEW und insbesondere Kalziumsulfat (Pflaster of Paris, Gips) ist von Natur aus hydrophil und gibt den Großteil der inkorporierten Wirkstoffe rasch ab, sodass der Retardeffekt gering ist [44]. Allerdings können verschiedene Kombinationen von Kalziumverbindungen im Trägermaterial (z. B. Kalziumsulfat mit -karbonat bzw. Kalziumverbindungen mit resorbierbaren Polymeren) oder die Beladung mit hydrophoben Antibiotikaverbindungen (z. B. einer Gentamicin-
Fettsäure-Verbindung) das Freisetzungsverhalten zudosierter Wirkstoffe positiv verändern [10]. Im letzteren Fall ist bei einer Beladung eines Trägermaterials aus Kalziumsulfat und -karbonat mit $1 \%$ Gentamicin-Palmitat eine retardierte hohe Freisetzung des Antibiotikums bis $\mathrm{zu} 72 \mathrm{~h}$ gezeigt worden. Eine erfolgreiche klinische Anwendung dieses synthetischen Knochenfüllmaterials im Rahmen der Infektsanierung und Knochenneubildung ist in mehreren klinischen Fallstudien dokumentiert worden [19, 47]. Ähnliches gilt auch für einen Gentamicin-beladenen injizierbaren KEW aus Hydroxyapatit und Kalziumsulfat [43].

\section{Kollagenschwämme}

Kollagenhaltige Wirkstoffträger sind ebenfalls wenig retardierte Trägersysteme, aus dem die angelagerten Antibiotika (kommerziell erhältlich nur Gentamicin) innerhalb weniger Stunden durch enzymatische Degradation des Kollagens rasch freigesetzt werden. Werden solche Trägersysteme mit einem hydrophilen Gentamicin-Salz ausgerüstet, fehlt jeglicher Retardeffekt. Durch Zugabe einer geringer löslichen Verbindung (z. B. Gentamicin-Crobefat) kann eine längere Freisetzungskinetik erreicht werden. Damit kann die Gentamicin-Ausscheidung für 2 bis 3 Tage sichergestellt werden [3].

Grundsätzlich gilt auch für diese Wirkstoffträger, dass der Zusatz hydrophober Substanzen und chemischer Additive, wie z. B. Polyethylenglycol (PEG), sowohl das Elutionsverhalten der zugesetzten Antibiotika als auch die Abbaugeschwindigkeit des Kollagens selbst merklich beeinflussen kann.

\section{Andere lokale Träger}

Polymere Nahtmaterialien [51] aus resorbierbaren (Milchsäure) oder nicht abbaubaren Materialien (Polyethylen, Polylactone) kommen ebenso als Träger für antiinfektive Substanzen in Betracht wie auch Polymertaschen für Herzschrittmacher [38, 64]. Prinzipiell können auch diese Implantate als Leitschiene für Keime fungieren, die sich mithilfe eines Biofilms auf der Oberfläche anhaften und dann in tiefere Körperregionen migrieren können.

\section{Antimikrobielle Beschichtungs- technologien}

Einen anderen Weg gehen spezielle Beschichtungstechnologien von Implantaten [24]. Dabei dienen die Implantate selbst als Wirkstoffträger, die ihre eigene Oberfläche schützen. Unterschieden wird zwischen passiver Oberflächenmodifikation, deren Ziel die Verhinderung einer Adhäsion von Bakterien ist, und der aktiven Beschichtung der Oberfläche mit antibakteriellen Wirkstoffen. Im letzteren Fall wird entweder die Strategie einer „Kontaktabtötung" verfolgt, wie im Falle der Inkorporation von ionischem oder Nanosilber auf der Oberfläche, oder die Strategie der kontrollierten aktiven Freisetzung von Antibiotika, Antiseptika oder antimikrobieller Peptide aus der Beschichtung, ähnlich wie im Falle antibiotikabeladener Knochenzemente [1, 38, 67]. Die Beschichtung kann entweder im industriellen Fertigungsprozess der Implantate kommerziell aufgesprüht werden oder auch perioperativ mit Hilfe eines speziell ausgerüsteten Gels erfolgen [62]. Zumindest im Fall von silberbeschichteten Tumorprothesen [66] bzw. eines Gentamicin-beschichteten Marknagels konnte die antimikrobielle Schutzwirkung und die mechanische Stabilität im klinischer Einsatz gezeigt werden $[25,65]$. Interessante antimikrobielle Beschichtungen stellen $D C^{\circledR}$, ein Gel aus einem Gemisch aus Hydroxyapatit (HA) und Poly-L-Laktid (PLLA [15]), PVPJod [32] und diverse Wirkstoff-Fettsäure-Gemische $[38,48,50,68]$ dar.

\section{PMMA als Wirkstoffträger}

Knochenzemente auf PMMA-Basis werden aus historischen, aber auch praktischen Gründen als Wirkstoffträger bevorzugt in der Endoprothetik eingesetzt [36], [6]. Ihre großen Vorteile sind die retardierte Freisetzung der eingeschlossenen Wirkstoffe, die lange klinische Erfahrung und die große Zahl an klinischen Studien. 
Unfallchirurg 2017· 120:561-572 DOI 10.1007/s00113-017-0372-8

(c) Der/die Autor(en) 2017. Dieser Artikel ist eine Open-Access-Publikation.

\section{K.-D. Kühn · N. Renz · A. Trampuz}

\section{Lokale Antibiotikatherapie}

\section{Zusammenfassung}

Behandlungen oberflächlicher Wundinfektionen mit lokalen Antibiotika werden wegen der erhöhten Gefahr einer Resistenzentwicklung und fehlender Wirksamkeit in tieferen Regionen als problematisch angesehen. Im Gegensatz dazu ist der lokale Einsatz von Antibiotika in der Knochenchirurgie bei Verwendung von Implantaten sinnvoll. Grund dafür ist die Gefahr einer raschen Ausbildung eines Biofilms auf körperfremden Implantaten. Eine daraus resultierende Infektion kann in der Folge unerkannt unterhalten werden und eine chronische Verlaufsform annehmen. Neben Fremdkörpern dient abgestorbenes Gewebe wie z. B. Knochensequester als Rückzugsgebiet für Keime im Körper. Aufgrund der Besonderheiten von Knochenund Gelenkinfektionen und der erheblichen Infektionsrezidivrate sind deshalb kombinierte Maßnahmen wichtig. Die systemische
Antibiotikagabe reduziert zwar die Anzahl der perioperativ eingebrachten bzw. hämatogen aus anderen Infektionsherden streuenden planktonischen Erreger, verfehlt aber aufgrund der geringen Knochengängigkeit vieler systemisch eingesetzten Antibiotika oftmals das Ziel, eine Besiedlung des Implantats zu verhindern. Lokal freigesetzte Antibiotika können dagegen aufgrund der hohen Konzentrationen in situ das körperfremde Material am Implantationsort vor Keimen schützen. Daher sind bei der Behandlung implantatassoziierter Infektionen systemisch und lokal applizierte antiinfektive Wirkstoffe als zuverlässige adjuvante Maßnahme in das Behandlungskonzept der chirurgischen Sanierung eingebettet, um den Therapieerfolg dauerhaft zu gewährleisten. Polymethylmethacrylat(PMMA)-Knochenzement ist dabei der am häufigsten verwendete lokale Wirkstoffträger. Mikrobiologen und Infektiologen bestimmen gemeinsam mit dem Chirurgen und Apotheker auf der Basis klinischer und mikrobiologischer Ergebnisse, welche antiinfektiven Wirkstoffe systemisch und welche mit PMMA-Zement lokal für den Patienten indiziert sind. Da im Gegensatz zu Empfehlungen für den systemischen Einsatz von Antiinfektiva für ihre lokale Applikation meist keine evidenten Konzepte existieren, wird in dem Übersichtsartikel nachfolgend dargestellt, welche Punkte hier beachtet werden sollten.

Schlüsselwörter

PMMA-Zement · Biofilm · Kolonisationsbarriere · Manuelle Zumischung · Empfehlungen

\section{Local antibiotic therapy}

\section{Abstract}

The treatment of superficial wound infections with local antibiotics is considered to be problematic as it is accompanied by an increased risk of resistance development and ineffectiveness in deeper regions. On the contrary, the local application of antibiotics is useful in arthroplasty and necessary if implants are utilized. The reason for this is the prevention of a rapid biofilm formation on foreign implants and a resulting infection may remain undiscovered until it becomes chronic. Apart from exogenous material, necrotic tissue (e. g. bone sequestra) provides suitable retreat areas for pathogens in the body. Because of the characteristics of bone and joint infections and an exceptional infection recurrence rate, a combined approach is required. Systemic antibiotic prophylaxis is able to reduce the number of pathogens acquired by perioperative contamination or hematogenic spread from another focus of infection; however, systemically applied antibiotics often fail to form an effective colonization barrier around the implant because their ability to penetrate the bone is relatively low. On the other hand, the high concentration of locally released antibiotics leads to an effective protection of the implant from bacteria in situ. Thus, for the treatment of implant-associated infections, systemic and local application of anti-infective agents is a reliable adjuvant measure that improves the therapeutic success. Polymethylmethacrylate (PMMA) bone cement is the most commonly used local drug carrier. Based on clinical and microbiological results, microbiologists and infectious disease experts together with the surgeon and pharmacist determine which anti-infective agents are indicated for systemic and local, PMMA cement-related application. Because there is no evident concept for the local application, unlike the recommendation for systemic use of anti-infective agents, this review article describes which aspects should be taken into account.

Keywords

PMMA cement - Biofilm - Colonization barrier . Manual application · Recommendations

\section{Medizinprodukte mit Antibiotika- zusatz}

PMMA-Knochenzemente sind per Definition Medizinprodukte und dienen in erster Linie der Verankerung künstlicher Gelenke im Körper. Vor Einführung des Medizinproduktegesetzes (MPG) im Jahre 1998 waren Knochenzemente mit und ohne Antibiotikazusatz Arzneimittel und nur einige wenige Knochen- zemente $\left(\right.$ Palacos $^{\circledR}$, Simplex $\left.^{\circledR}, \mathrm{CMW}^{\circledR}\right)$ erfüllten die hohen Standards der Arzneimittelbehörden. Diese sogenannten Altspezialitäten sind auch heute noch in unveränderter Qualität im Markt erhältlich und deren klinische Evidenz ist in vielen Studien und Fallbeispielen belegt. Dabei wird Palacos ${ }^{\circledR}$, der im deutschsprachigen Raum im Jahr 1958 eingeführt wurde, nach einer Literaturrecherche der Jason Consulting in den meisten publizierten klinischen Studien als Verankerungsmaterial verwendet [37].

\section{》) Die sog. Altspezialitäten sind heute noch in unveränderter Qualität erhältlich}

Der Antibiotikazusatz im Knochenzement ändert an der ursprünglichen 


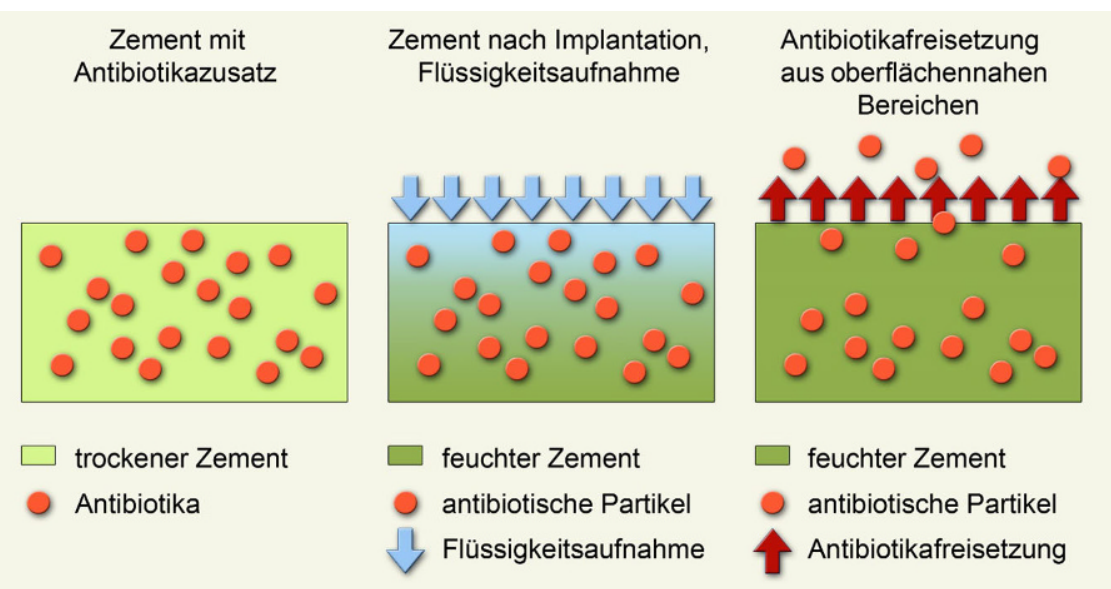

Abb. $1 \Delta$ Diffusionsmodell für Antibiotika aus Polymethylmethacrylat(PMMA)-Knochenzement nach Implantation und Aufnahme von Körperflüssigkeiten

Zweckbestimmung des Medizinproduktes nichts. Allerdings erhält dieses Biomaterial neben seiner Hauptfunktion als Verankerungsmaterial die zusätzliche Aufgabe, das körperfremde Material vor einer Besiedelung durch Keime zu schützen und gleichzeitig durch aktive Antibiotikafreisetzung die Anzahl möglicher Keime im Operationsfeld lokal zu reduzieren.

Die Antibiotika im PMMA-Zement übernehmen somit 2 Funktionen: die einer Kolonisationsbarriere direkt an der Zementoberfläche und die einer adjuvanten therapieunterstützenden keimreduzierenden Wirkung im Knochen, Weichteil und Sekret. Ein weiterer großer Vorteil des Einsatzes antibiotikabeladener Zemente ist, dass selbst in Bezug auf Nebenwirkungen problematische Antiinfektiva in relativ hohen Konzentrationen an den gewünschten Ort gebracht werden können, ohne dass dabei systemisch hohe oder gar toxische Wirkspiegel aufgebaut werden.

\section{Wirkstofffreisetzung durch Diffusion}

Das Prinzip der lokalen Antibiotikagabe wurde durch Prof. Buchholz und seinen Mikrobiologen Dr. Lodenkämper in Hamburg am Beispiel des Gentamicinhaltigen Palacos ${ }^{\circledast}$ R erstmals untersucht. Das Herauslösen mobiler, nicht in den Polymerketten der Zementmatrix eingebundenen Substanzen, ist die physikalische Grundlage für die Abgabe von Wirkstoffen aus Knochenzement. Dies ist eine
Frage der Oberfläche, d.h. die Freisetzung folgt den Gesetzen der Diffusion, die eng verbunden ist mit der Wasseraufnahmefähigkeit des PMMA-Zements (• Abb. 1). Die Wirkstofffreisetzung verhält sich einerseits proportional zur zeitlichen Wasseraufnahmefähigkeit des Zements und andererseits zur vorhandenen Zementoberfläche [7].

\section{Gentamicin-haltiges Palacos ${ }^{\circledR} \mathrm{R}$}

Als Basis aller pharmakokinetischen Untersuchungen zu Knochenzementen gilt das Elutionsverhalten des Gentamicinhaltigen Palacos ${ }^{\circledast}$ R nach Wahlig [69]. Im spongiösen Knochengewebe in Nachbarschaft zum Knochenzement waren Werte des Antibiotikums von bis $\mathrm{zu} 47 \mu \mathrm{g} / \mathrm{ml}$ nachweisbar, im Bindegewebe von bis zu $17 \mu \mathrm{g} / \mathrm{ml}$ und selbst im kortikalen Knochen noch $1 \mu \mathrm{g} / \mathrm{ml}$. Systemisch waren die gemessenen Gentamicin-Konzentrationen dagegen sehr gering (Serum $0,7 \mu \mathrm{g} / \mathrm{ml}$, Urin $17 \mu \mathrm{g} / \mathrm{ml}$ - nach $24 \mathrm{~h}$ ). Im Vergleich dazu konnte bei systemischer Gentamicin-Applikation (Einmalinjektion von einmal $80 \mathrm{mg}$ i.m. GentamicinSulfat-Lösung) das Gegenteil beobachtet werden: geringe lokale Antibiotikakonzentration von nur $0,4 \mu \mathrm{g} / \mathrm{ml}$ und hohe Spiegel im Serum von $5 \mu \mathrm{g} / \mathrm{ml}$ und im Urin von $200 \mu \mathrm{g} / \mathrm{ml}$ (• Abb. 2).

\section{Beeinflussung der Freisetzung}

Eine Vielzahl von Faktoren beeinflusst die Freisetzung der Wirkstoffe aus PMMA. Unmittelbar bei Implantation wird bereits ein antibiotischer Film auf der

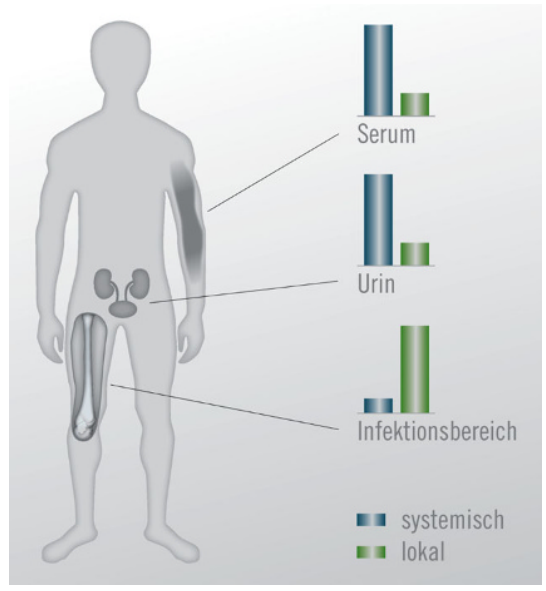

Abb. 2 \ Vergleich der Antibiotikakonzentrationen im Serum, Urin und Infektionsbereich nach systemischer Gabe von einmal $80 \mathrm{mg}$ Gentamicin i.m. und lokaler Gabe von $1,25 \%$ Gentamicin im Polymethylmethacrylat(PMMA)-Zement. (Nach Wahlig [69], aus [37])

Zementoberfläche ausgebildet, der als Kolonisationsbarriere über längere Zeit bestehen bleibt, da aus tieferen Schichten des PMMA weitere Wirkstoffpartikel per Diffusion herausgelöst werden. Je höher der Wirkstoffgehalt im PMMA, desto besser ist auch die Freisetzung. Eine Wirkstoffzugabe zum PMMA hat aber Grenzen: Mit steigender Konzentration des zugemischten Wirkstoffs wird der PMMA-Zement immer inhomogener und die mechanische Stabilität verschlechtert sich signifikant. Es sollte auch darauf hingewiesen sein, dass die $\mathrm{Zu}$ mischung hoher Dosen eines Wirkstoffs zum PMMA das Produkt dahingehend verändert, das es als nichtkonformes Medizinprodukt eingesetzt wird. Generell gilt: Je größer die Zementoberfläche, desto höher ist die freisetzende Fläche und die Elutionsrate [69].

》) Je größer die Zementoberfläche, desto höher die freisetzende Fläche und die Elutionsrate

Diffusionsprozesse sind zudem beeinflussbar durch Hydrophilie. PMMAZemente mit hydrophilen Polymeren bzw. Kopolymeren haben eine erhöhte Fähigkeit, Körperflüssigkeiten aufzunehmen und dadurch Wirkstoffe besser aus der Zementmatrix herauszulösen. 
Tab. 1 Antibiotika, diein Kombination mit Polymethylmethacrylat(PMMA)-Zementen erhältlich sind [40]

\begin{tabular}{|c|c|c|c|}
\hline Antibiotikum & $\begin{array}{l}\text { Antibiotikaklasse und } \\
\text { Wirkungsmechanis- } \\
\text { mus }\end{array}$ & Wirkungsspektrum & Beispiel \\
\hline $\begin{array}{l}\text { Gentamicin } \\
1,25-2,5 \%\end{array}$ & $\begin{array}{l}\text { Aminoglykosid } \\
\text { Bakterizid } \\
\text { Hemmt die Protein- } \\
\text { synthese und bildet } \\
\text { Nonsenseproteine, } \\
\text { bindet am 30S-Ribosom }\end{array}$ & $\begin{array}{l}\text { Breit wirksam gegen } \\
\text { viele grampositive } \\
\text { und -negative Bakte- } \\
\text { rien (Staphylococcus } \\
\text { spp., Enterococcus } \\
\text { spp., Pseudomonas, } \\
\text { E. coli, Klebsiella, Pro- } \\
\text { teus) }\end{array}$ & Palacos R + G \\
\hline Tobramycin 2,5\% & $\begin{array}{l}\text { Aminoglykosid } \\
\text { Bakterizid } \\
\text { Hemmt die Protein- } \\
\text { synthese und bildet } \\
\text { Nonsenseproteine, } \\
\text { bindet am 30S-Ribosom }\end{array}$ & Wie bei Gentamicin & $\begin{array}{l}\text { Simplex }{ }^{\circledR} \text { mit Tobra- } \\
\text { mycin }\end{array}$ \\
\hline Clindamycin 2,5\% & $\begin{array}{l}\text { (Lincoamid) } \\
\text { Bakteriostatisch (in } \\
\text { hohen Konzentratio- } \\
\text { nen auch bakterizid); } \\
\text { hemmt die Protein- } \\
\text { synthese, bindet am } \\
\text { 50S-Ribosom }\end{array}$ & $\begin{array}{l}\text { Wirkt gegen viele } \\
\text { grampositive und } \\
\text {-negative Bakterien } \\
\text { (Streptococcus spp., } \\
\text { Staphylococcus spp., } \\
\text { Anaerobier, z. B. } \\
\text { Propionibacterium } \\
\text { acnes) }\end{array}$ & $\mathrm{Copa}^{\circledR} \mathrm{G}+\mathrm{C}$ \\
\hline Vancomycin 2,5-5\% & $\begin{array}{l}\text { (Glykopeptid) } \\
\text { Bakterizid } \\
\text { Hemmung der Zell- } \\
\text { wandsynthese }\end{array}$ & $\begin{array}{l}\text { Wirkt gegen viele } \\
\text { grampositive Bakte- } \\
\text { rien (Staphylococcus } \\
\text { spp., inklusive MRSA } \\
\text { und MRSE, Enterococ- } \\
\text { cus spp.) }\end{array}$ & $\mathrm{Copal}^{\circledR} \mathrm{G}+\mathrm{V}$ \\
\hline Erythromycin 1,25\% & $\begin{array}{l}\text { (Makrolid) } \\
\text { Bakteriostatisch, } \\
\text { hemmt die Protein- } \\
\text { synthese am Ribosom }\end{array}$ & $\begin{array}{l}\text { Wirkt gegen viele } \\
\text { grampositive (Strep- } \\
\text { tococcus spp., Sta- } \\
\text { phylococcus spp.), } \\
\text { Anaerobier (Coryne- } \\
\text { bacteria) }\end{array}$ & $\begin{array}{l}\text { Simplex }{ }^{\circledR} \text { mit Ery- } \\
\text { thromycin und Colis- } \\
\text { tin }\end{array}$ \\
\hline Colistin 0,6\% & $\begin{array}{l}\text { (Polypetid) } \\
\text { Bakterizid, verändert } \\
\text { die Permeabilität der } \\
\text { Zytoplasmamembran } \\
\text { (Plasma läuft aus) }\end{array}$ & $\begin{array}{l}\text { Wirkt gegen gramne- } \\
\text { gative Bakterien } \\
\text { (Pseudomonas aerugi- } \\
\text { nosa, Klebsiella spp., } \\
\text { Acinetobacter app.) }\end{array}$ & $\begin{array}{l}\text { Simplex }{ }^{\circledR} \text { mit Ery- } \\
\text { thromycin und Colis- } \\
\text { tin }\end{array}$ \\
\hline
\end{tabular}

Eine zu hohe Hydrophilie führt allerdings zur Quellung. Die Zementviskosität hat ebenfalls einen Einfluss auf die Freisetzung. Je unbeweglicher die Polymerketten werden, desto geringer ist die Diffusion. Niedrigviskose Zemente mit kurzer Verarbeitungsbreite werden in einem kürzeren Applikationsfenster implantiert, während hochviskose PMMAZemente nach Implantation noch länger viskös bleiben, bis die komplette Aushärtung erfolgt. In der viskosen Phase tran, Xylit), die die Porosität der Zementmatrix erhöhen sollen, können die Freisetzung der Wirkstoffe aus PMMA deutlich erhöhen. Bei Kombination mancher antiinfektiver Wirkstoffe sind zudem synergistische Effekte in Bezug auf das Freisetzungsverhalten nachgewiesen worden. So ist z. B. die gegenseitige Wirkstoffelution bei Kombination von Gentamicin + Vancomycin oder Gentamicin + Clindamycin besonders stark erhöht [40]. Grundsätzlich gilt, dass bei Zumischung antiinfektiver Wirkstoffe immer auch mögliche Wechselwirkungen mit Bestandteilen des PMMA und der chemischen Reaktion (radikalische Polymerisation, cave: exotherme Wärmeentwicklung) in Betracht gezogen werden müssen.

PMMA-Zement mit Antibiotikazusatz wird in erster Linie zur Gelenkverankerung in der primären und in der Revisionsendoprothetik eingesetzt. Die örtlich in hohen Konzentrationen freigesetzten Antibiotika dienen in niedrigerer Dosis (0,5-1\% Antibiotikum im Zementpulver) der Prävention einer periprothetischen Infektion, in höherer Dosis (5-10\% Antibiotikum im Zementpulver) der Therapieunterstützung des chirurgischen Débridements in Kombination mit systemisch verabreichten Antibiotika [26-28, 59]. Wegen der limitierten Penetration der systemisch verabreichten Antibiotika in die schwer zugänglichen Kompartimente, wie Knochen oder Gelenke, macht es bei orthopädischen/unfallchirurgischen Operationen mit hohem Infektionsrisiko Sinn, zusätzlich lokale Wirkstoffträger an den Ort einer möglichen Kontamination $\mathrm{zu}$ bringen. In der Endoprothetik hat sich daher die kombinierte Antibiotikagabe aus systemischer und lokaler Gabe im PMMA-Zement durchgesetzt, deren Evidenz sich u. a. durch Registerdaten bestätigen lässt. Es ist jedoch weiterhin umstritten, ob bei der primären Implantation bei Hochrisikopatienten ein antibiotikahaltiger Zement routinemäßig eingesetzt werden soll.

\section{Manuelle Zumischung}

Die Zumischung von Antibiotika ist immer dann notwendig und sinnvoll, wenn 


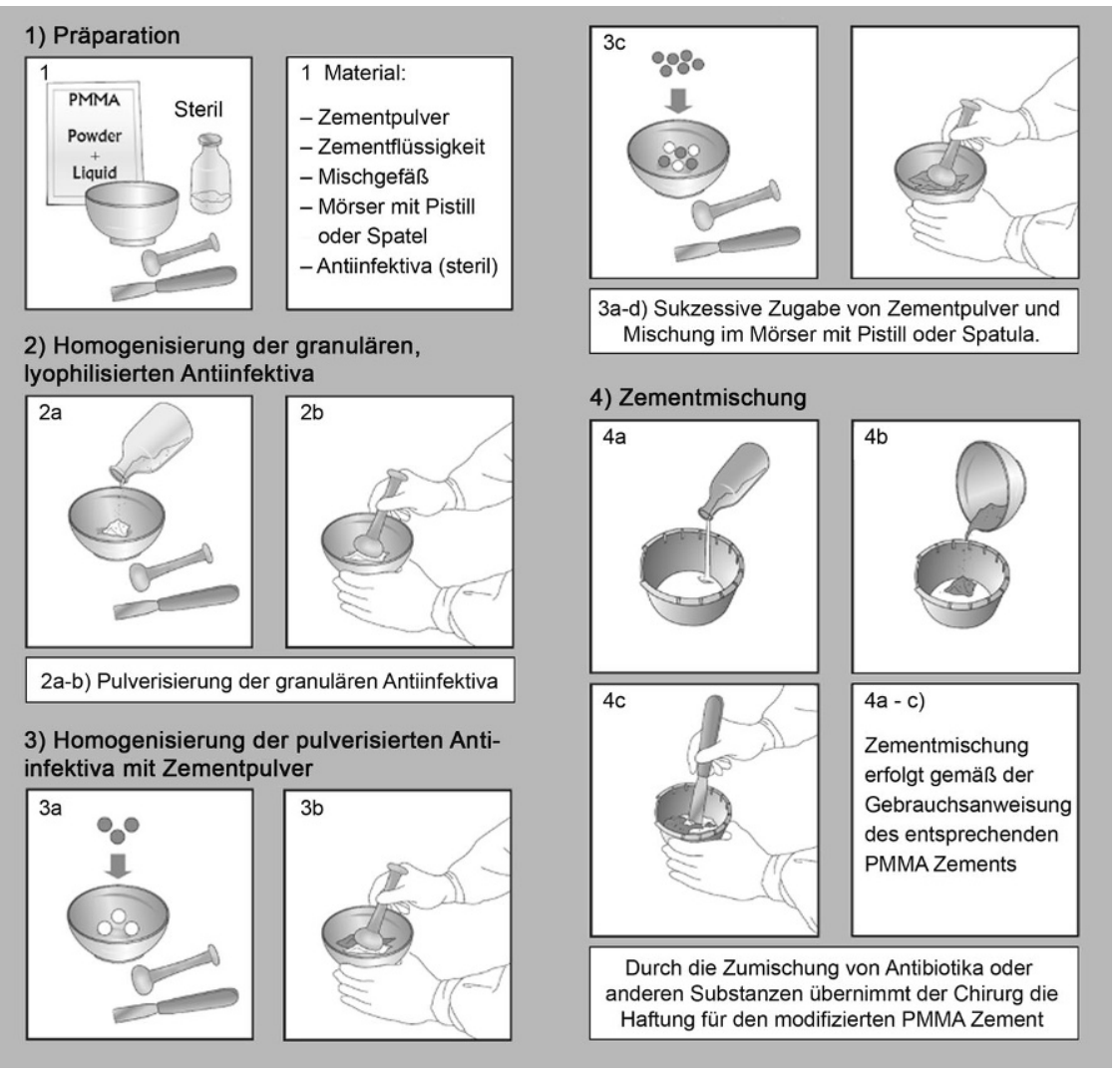

Abb. 3 A Anleitung zum Zumischen von Antiinfektiva zu Polymethylmethacrylat(PMMA)-Zementpulver nach Kühn [37], verändert nach Ochsner et al. [52]. (Aus [37])

keine handelsüblichen Knochenzemente mit den für die jeweilige Operation benötigten Antibiotika erhältlich sind. Die Zumischung der Antibiotika erfolgt in der Regel perioperativ während der Zementherstellung. Eine manuelle $\mathrm{Zu}-$ mischung antiinfektiver Wirkstoffe kann immer dann indiziert sein, wenn folgende Voraussetzungen vorliegen:

- die detektierten Keime werden nicht durch das Keimspektrum der in der - Tab. 1 aufgeführten kommerziell erhältlichen antibiotikahaltigen Zemente abgedeckt,

- die Menge an Antibiotika in den vorliegenden PMMA-Zementen wird als nicht ausreichend empfunden und soll manuell erhöht werden,

- bei Risikopatienten mit Vorerkrankungen reicht das antimikrobielle Spektrum nicht aus und muss zielgerichtet erweitert werden,

- die Freisetzung der Antibiotika im Zement ist ungünstig und niedrig, insbesondere bei einer Spaceranwendung über mehrere Wochen,
- es bestehen Überempfindlichkeiten gegen bestimmte Antibiotika.

In derzeit handelsüblichen PMMA-Zementen finden sich lediglich 6 industriell zugemischte Antibiotika (•Tab. 1), die entweder alleine oder in Kombination zur Verfügung stehen.

Als Obergrenze für das nachträgliche manuelle Zumischen werden $4 \mathrm{~g}$ Antibiotika auf $40 \mathrm{~g}$ Zementpulver empfohlen (10\%-Regel [21, 26, 41, 42]).

\section{》) Für das Zumischen werden als Obergrenze $4 \mathrm{~g}$ Antibiotika auf $40 \mathrm{~g}$ Zementpulver empfohlen}

Von einer Zumischung flüssiger Antibiotika wird abgeraten, weil insbesondere die mechanische Stabilität erheblich beeinflusst werden kann [22]. Allerdings kann es in seltenen Fällen bei Nichtverfügbarkeit steriler Wirkstoffe in Pulverform notwendig sein, doch auf ein flüssiges Antibiotikum zurückzugreifen. Für diesen
Fall existieren aber keine Vorgaben, wie und in welchen Konzentrationen das flüssige Antibiotikum zu dosiert werden soll $[8,29]$.Zudem ist nicht bekannt, welchen Einfluss eine solche Zumischung auf die Qualität des PMMA-Zementes hat.

Bei Augmentationen werden sogar Zytostatika dem PMMA-Zement zugemischt und anschließend in den Knochendefekt eingebracht [39]. Werden antiinfektive Substanzen einem zugelassenen Produkt manuell zugegeben, gilt derjenige, der das Produkt verändert hat, als Hersteller im Sinne des Medizinproduktegesetzes und trägt alle Haftungsrisiken selbst.

\section{Beispiel Vancomycin-Zumischung}

In der klinischen Praxis wird insbesondere Vancomycin dem Knochenzement von Hand zugemischt, obwohl zertifizierte Vancomycin-haltige Produkte im Markt erhältlich sind. Auf jeden Fall sollte man bei manueller Zumischung von Vancomycin Folgendes beachten:

- Ist das Vancomycin steril?

- Welche Konsistenz weist Vancomycin auf?

- In welcher Salzverbindung wird das Vancomycin geliefert (Hydrochlorid)?

- Welchem PMMA-Zement soll das Vancomycin zugemischt werden?

Steriles Vancomycin kann relativ leicht von der Klinikapotheke bezogen werden. Allerdings gilt dies nicht für alle erhältlichen Antibiotika. Werden die Substanzen in Ampullen geliefert, sollten diese nie über dem Anmischgefäß geöffnet werden, um Glassplitter im Gefäß zu vermeiden. Da bestimmte Antibiotika in manchen Ländern nur in flüssiger Form steril erhältlich sind, muss bei Zumischung der Flüssigkeit in Betracht gezogen werden, dass insbesondere die mechanischen Eigenschaften von Knochenzement negativ beeinflusst werden $[8,20]$. Des Weiteren sollte beachtet werden, dass Vancomycin in unterschiedlichen Qualitäten erhältlich ist: lyophylisiertes Vancomycin enthält grobe Partikel, die sich durch einfaches Mischen nicht zerkleinern lassen. In diesem Fall sollte Vancomycin vorab mit einem Mörser zerkleinert und erst dann mit dem Zementpulver vermischt 


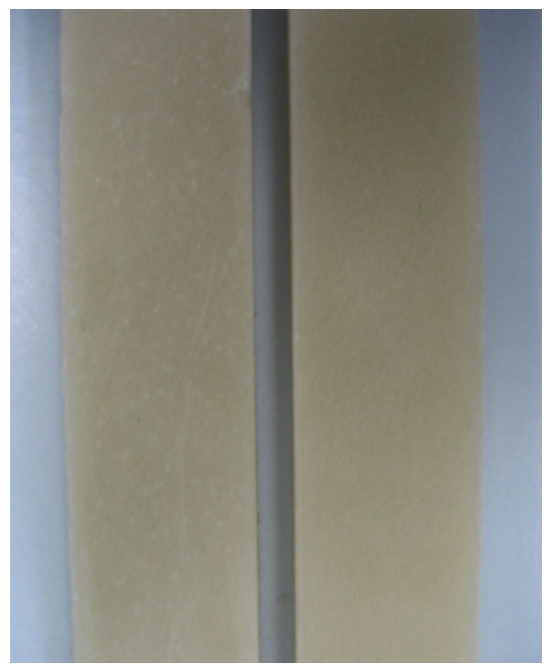

Abb. 4 ム ISO-Formkörper aus Polymethylmethacrylat(PMMA)-Zement: Inhomogene manuelle Zumischung von Vancomycin (links) mit Stippen im Vergleich zur industriellen Vancomycin-Zudosierung (rechts) ohne Stippen

werden. Wenn keine Zerkleinerung erfolgt, wird Vancomycin nicht homogen in den Zement eingemischt mit der Folge, dass die Freisetzung, die Handlingeigenschaften und die mechanische Stabilität des Knochenzements variieren können.

Eine weitere Unbekannte für viele Chirurgen ist die Unterscheidung von aktiver und Gesamtwirksubstanz. So besteht Vancomycin-Hydrochlorid nur aus $93 \%$ aktiver Substanz, d. h. $7 \%$ des eingesetzten Vancomycins sind unwirksam. Werden demnach $2 \mathrm{~g}$ Vancomycin dem Knochenzement zugemischt, sind davon nur 1,86 g aktiv. Beim Gentamicin wird das noch deutlicher: werden $2 \mathrm{~g}$ Gentamicin-Sulfat eingesetzt, sind im Zement lediglich ca. 1,2 g aktive Gentamicinbase vorhanden. Dies ist insbesondere deshalb von Bedeutung, weil lediglich ca. $10 \%$ des im Zement befindlichen Wirkstoffs tatsächlich freigesetzt werden und die restliche Menge in der Zementmatrix verbleibt.

\section{Reproduzierbarkeit der Zumischung}

Reproduzierbare Wirkstofffreisetzungen sind nur möglich, wenn Antibiotika im PMMA-Zement homogen verteilt sind. Inhomogenitäten sind daher unbedingt zu vermeiden. Beim Zumischen von weißem Antibiotika- zu weißem Zementpulver sind grobe Antibiotika-

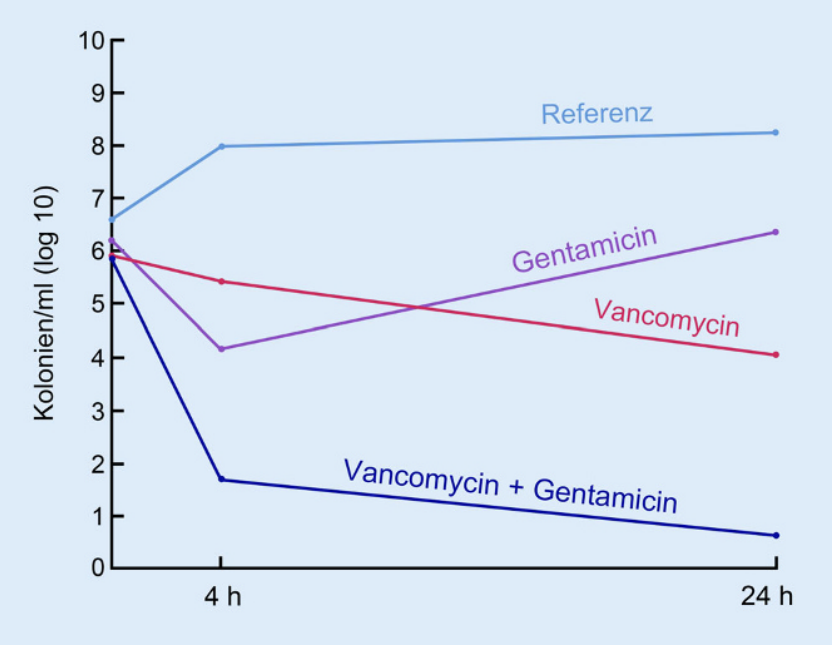

Abb. $5<$ Abtötungsraten eines Gentamicin-resistenten Methicillin-resistenten Staphylococcus aureus (MRSA), eines Vancomycin-empfindlichen MRSA und einer Kombination beider Antibiotika zur Darstellung des synergistischen Effekts [46] partikel kaum auszumachen. Werden dagegen solche Antibiotika mit grünem Zementpulver vermischt, sind grobe Antibiotikapartikel leicht erkennbar. Um homogene Mischungen zu erhalten, wird empfohlen, Antiinfektiva unter Zuhilfenahme einer Zumischanleitung (• Abb. 3) dem PMMA-Pulver zuzugeben $[23,52]$.

Manche antiinfektiven Wirkstoffe können wegen chemischer Wechselwirkungen den PMMA-Zement farblich verändern und nach Aushärtung durchaus eine andere als üblicherweise gewohnte Farbe aufweisen.

\section{Inhomogenitäten nach Zumischung}

Bisweilen wird argumentiert, dass bei Anwendung eines antibiotikahaltigen Knochenzements im Spacer die mechanische Stabilität von untergeordneter Bedeutung ist. Auch wenn die Verweilzeit eines solchen Spacers mit i.d. R. 4 bis 8 Wochen limitiert ist, ist diese Sichtweise wissenschaftlich und rechtlich durchaus problematisch:

- Grundlage einer Zulassung von Knochenzement ist die Erfüllung von festgelegten Normen, z. B. in Bezug auf mechanische Stabilitätstestungen (ISO 5833, [30]). Wird ein Produkt manuell verändert (insbesondere Inhomogenitäten der zugemischten Antiinfektiva; - Abb. 4), können Normwerte unterschritten werden, zudem wird ein nicht gesetzeskonformes Produkt eingesetzt.
- Wirkstoffpartikel im Zement führen nicht zwangsläufig zu schwächeren mechanischen Festigkeitswerten. Eine erhöhte Hygrophilie kann durchaus ein höheres Elastizitätsmodul oder Druckfestigkeit führen, während die Biegefestigkeit nicht mehr ISO-konform ist.

- Inhomogen verteilte Wirkstoffe reduzieren nicht nur die mechanische Stabilität, es können ggf. Zementpartikel leichter aus der Matrix brechen.

- Inhomogen verteilte Wirkstoffpartikel in der Zementmatrix (Stippen, - Abb. 4), können die Freisetzung der Wirkstoffe signifikant erniedrigen oder erhöhen mit der Folge, dass die Freisetzung unkontrolliert erfolgt.

- Ein unkontrolliertes Elutionsverhalten kann zu kurzzeitigen toxischen Wirkspiegeln führen und/oder nicht ausreichend lang protektiv wirken. Im schlimmsten Fall kann dies zu einer bakteriellen Wiederbesiedelung des Spacers führen, bevor alle noch verbliebenen Bakterien abgetötet sind.

Eine Zumischung von Antibiotika zum PMMA-Pulver vor der Zementherstellung ist die klassische und sichere Methode, eine Zugabe von Vancomycin auf die Oberfläche eines noch nicht ausgehärteten Spacers wird ebenfalls diskutiert. Dabei scheint die Gefahr einer erhöhten Antibiotikakonzentration nach Zugabe von 1 bzw. $2 \mathrm{~g}$ Vancomycin auf den Zementteig sowohl systemisch ebenso un- 
Tab. 2 Empfehlungen der PRO-IMPLANT-Foundation vom 23. Januar 2017 (Version 5) für lokale Antiinfektiva in Polymethylmethacrylat(PMMA)-Zement zusätzlich zur chirurgischen und systemischen Therapie [61]

(1)

Enterobacteriaceae

Aminoglykosid-S

ESBL-Bildner oder Aminoglykosid-R

Carbapenem-R oder Aminoglykosid-R

Nonfermenter

Aminoglykosid-S und Chinolon-S

Multi-R

Anaerobier

Candida spp.

\section{Antibiotikum}

Gentamicin +

Clindamycin

Gentamicin +

Daptomycin oder

Vancomycin

Gentamicin +

Clindamycin

Vancomycin

Gentamicin +

Linezolid oder

Daptomycin oder

Fosfomycin

Gentamicin

(+/- Clindamycin)

Gentamicin +

Meropenem

Gentamicin +

Colistin

\begin{tabular}{l}
$\begin{array}{l}\text { Mikroorganismus ( } \mathbf{S}=\text { sens } \\
\boldsymbol{R}=\text { resistent) }\end{array}$ \\
\hline $\begin{array}{l}\text { Staphylococcus spp. } \\
\text { Oxacillin-/methicillin-S } \\
\text { Oxacillin-/methicillin- } \boldsymbol{R}\end{array}$ \\
Streptococcus spp. \\
Enterococcus spp. \\
Vancomycin-S \\
Aminoglykosid-S oder - $\boldsymbol{R}$ \\
Vancomycin- $\boldsymbol{R} /$ \\
Aminoglykosid-S oder - $\boldsymbol{R}$
\end{tabular}

Gentamicin +

$\begin{array}{ll}\text { Gentamicin + } & 0,5 \\ \text { Ciprofloxacin } & 2 \\ \text { Gentamicin + } & 0,5 \\ \text { Colistin oder } & 1-2 \\ \text { Fosfomycin } & 1-2 \\ \text { Gentamicin + } & 1 \\ \text { Clindamycin } & 1 \\ \text { Gentamicin + } & 0,5 \\ \text { Amphotericin B liposomal } & 0,2-0,3 \\ \text { (Ambisome) oder } & 0,2-0,8 \\ \text { Amphotericin B nicht-liposo- } & 0,3-0,6 \\ \text { mal (Fungizone) oder } & \\ \text { Voriconazol } & \end{array}$

begründet zu sein wie die einer lokalen Reaktion durch extrem hohe Vancomycin-Spiegel im Wundsekret $[2,5]$.

\section{Synergien}

Die Zementbasis als Wirkstoffträger spielt insbesondere bei Revisionen eine erhebliche Rolle. Grundsätzlich ist $\mathrm{zu}$ empfehlen, stets einen antibiotikahaltigen Zement als Basis für die Zumischung $\mathrm{zu}$ verwenden anstatt eines antibiotikafreien Zements. Hauptgrund dafür ist die sich häufig einstellende Synergie im Freisetzungsverhalten, d. h. eine verbesserte Elution des zugemischten und des bereits im Zement befindlichen Antibio- tikums. Klassisches Beispiel dafür ist die Kombination aus Gentamicin und Vancomycin. Dabei gilt, je hydrophiler die Zementbasis und je besser bereits das Freisetzungsverhalten des antibiotikahaltigen Basiszementes ist, desto besser wird auch das manuell zugemischte Antibiotikum aus der Oberfläche des Zementmantels eluieren. Physikalisch ist dies erklärbar durch die höhere Kapazität der Flüssigkeitsaufnahme und die damit verbundene verbesserte Diffusion (• Abb. 1).

Synergien in Bezug auf eine stärkere antimikrobielle Wirksamkeit sind ebenfalls für manche Antibiotikakombinationen beschrieben worden. Am
Beispiel von Gentamicin in Kombination mit Vancomycin hat sich gezeigt, dass dieser beobachtete Effekt gegen MRSA bzw. Penicillin-resistente Pneumokokken möglicherweise auf eine vermehrte intrazelluläre Penetration von Gentamicin zurückzuführen ist, nachdem Vancomycin die Permeabilität der Zellwand erhöht hat. Die Abtötungsrate (• Abb. 5) von einer Mischung aus Gentamicin und Vancomycin ist im Vergleich zu der der jeweiligen Antibiotika alleine signifikant $[13,46]$.

\section{》) Gentamicin plus Vancomycin sind im Vergleich zum Antibiotikum alleine signifikant wirksamer}

Physikalische und chemische Unterschiede bei verschiedenen PMMA-Zementen führen $\mathrm{zu}$ unterschiedlichem Elutionsverhalten: die gewählte Zementbasis ist daher enorm wichtig. Berichte über Zumischungen und den Einfluss auf Freisetzung und Mechanik sind daher nur bedingt von einem Zement auf den anderen übertragbar. Klinische Studien haben gezeigt, dass eine $\mathrm{Zu}$ mischung z.B. von Vancomycin zum gut freisetzenden Zement Copal G + C (enthält bereits je $1 \mathrm{~g}$ Gentamicin und Clindamycin) eine hervorragende Option für einen sehr breit wirkenden und gut freisetzenden Spacer sein kann. So wurden im angrenzenden Gewebe hohe Antibiotikakonzentrationen für alle 3 Wirkstoffe deutlich oberhalb der minimalen Hemmkonzentration (MHK) noch 4 Wochen über die Spacerverweildauer hinaus nachgewiesen [17].

\section{Zumischempfehlungen nach Keimstatus}

Die größte Aussicht auf Behandlungserfolg bei Knochen- und Gelenkinfektionen besteht dann, wenn das Behandlungsteam eine Vielzahl von Faktoren in ihre Betrachtung einbezieht. Darunter gehören u. a. Patientenanamnese, Analyse der Knochenqualität, Kenntnis des Keims und dessen antibiotische Resistenzen sowie das Zusammenspiel und 
Tab. 3 Alternativen bei Überempfindlichkeiten gegen bestimmte Antibiotika unter Verwendung von Polymethylmethacrylat(PMMA)-Zement mit und ohne manuelle Zumischung

\begin{tabular}{|c|c|c|}
\hline Allergie gegen & Keimnachweis auf & AB-Alternative \\
\hline \multicolumn{3}{|l|}{ Aminoglykosid } \\
\hline Gentamicin/Tobramycin & Staphylokokken & Clindamycin \\
\hline \multicolumn{3}{|l|}{ Aminoglykosid/Lincosamid } \\
\hline $\begin{array}{l}\text { Gentamicin + Clindamycin } \\
(G+C)\end{array}$ & $\begin{array}{l}\text { Staphylokokken, Propioni- } \\
\text { bakterien }\end{array}$ & $\begin{array}{l}\text { Colistin, Daptomycin, Linezolid, } \\
\text { Cefuroxim }\end{array}$ \\
\hline \multicolumn{3}{|l|}{ Glykopeptid } \\
\hline Vancomycin/Teicoplanin & MRSA & Linezolid, Daptomycin, Chinolone \\
\hline \multicolumn{3}{|l|}{ Polymyxin } \\
\hline Colistin & $\begin{array}{l}\text { Pseudomonas, Propionibak- } \\
\text { terien }\end{array}$ & Gentamicin + Clindamycin $(G+C)$ \\
\hline \multicolumn{3}{|l|}{ Makrolid } \\
\hline Erythromycin & Staphylokokken & Aminoglykoside, $\mathrm{G}+\mathrm{C}$ \\
\hline \multicolumn{3}{|c|}{ MRSA Methicillin-resistenter Staphylococcus aureus } \\
\hline
\end{tabular}

die Optimierung der systemischen und lokalen Antibiotikagabe. Aus - Tab. 1 geht hervor, welche Antibiotika mit PMMA in Kombination bereits industriell vorgemischt vorliegen. Bei der Behandlung „schwieriger“ grampositiver Bakterien, bei denen eine reduzierte Empfindlichkeit oder gar eine Vancomycin-Resistenz vorliegt, können je nach Antibiogramm lokal auch Fosfomycin, Fusidinsäure, Levofloxacin, Linezolid, Daptomycin oder Teicoplanin notwendig werden. Bei gramnegativen multiresistenten Keimen können Meropenem, Fosfomycin, Colistin oder Amikacin zur lokalen Anwendung ratsam sein. Auch bei Mischinfektionen, die immer häufiger diagnostiziert werden, ist eine sehr breite Abdeckung durch Zumischung von Antiinfektiva relevant. Für die Bekämpfung von Candida-Infektionen steht $u$. a. das liposomale Amphotericin B zur Zumischung zum Zement zur Verfügung. Alle die zuvor genannten antiinfektiven Substanzen sind gut mit PMMAZement kombinierbar und sollten dann zugemischt werden, wenn handelsübliche antibiotikahaltige Knochenzemente nicht mehr ausreichend sind [40]. Die in -Tab. 2 genannten Empfehlungen geben einen Überblick, welche antiinfektiven Substanzen in welcher Dosierung keimgerecht zum Knochenzement zugemischt werden können, um die systemische Antibiotikatherapie nach zuvor erfolgtem chirurgischem Débridement zu komplettieren.
Die oben genannten Empfehlungen [61] basieren auf nachfolgenden Erkenntnissen und sollten beachtet werden:

- Die Bestimmung der MHK orientiert sich an systemisch zu erzielenden Antibiotikakonzentrationen. Wegen oft wesentlich höherer lokaler Konzentrationen und synergistischer Effekte sind sie nicht 1:1 auf die lokale Applikation übertragbar.

- Nebenwirkungen und Interaktionen lokaler Antibiotika sind selten, dennoch müssen Serumspiegel von Vancomycin und von Aminoglykosiden (Gentamicin) bei Niereninsuffizienz (geschätzte glomeruläre Filtrationsrate $[\mathrm{eGFR}]<60 \mathrm{ml} / \mathrm{min}$ ) und gleichzeitiger systemischer Applikation überwacht werden.

- Nur pulverförmige Antibiotika und keine flüssigen Substanzen verwenden! Antibiotika, die die Polymerisation hemmen (wie z. B. Rifampicin, Imipenem oder Metronidazol) oder die nicht thermostabil sind ( $\mathrm{z}$. B. gewisse Betalaktame) sollten nicht verwendet werden.

- Minimale effektive Dosierung. Vor allem zur Fixierung von Implantaten sollte die maximal empfohlene Dosierung $10 \%$ der Pulvermenge nicht überschreiten.

- Die PIF-Empfehlungen basieren auf der Erfahrungslage zu Palacos ${ }^{\circledR} /$ $\mathrm{Copal}^{\circledR}$-Knochenzementen sowie auf Literaturdaten.
- Im Vergleich zu den industriell gefertigten Produkten kann die manuelle Zumischung von Antibiotika in den Zement zu einer geringeren mechanischen Stabilität und geringeren Freisetzung führen. Bewertungen der mechanischen Kennwerte wurden gemäß ISO 5833 [30] vorgenommen; Legende: $(++)=$ registriertes Produkt; $(+)=$ Erfüllung der ISONorm wahrscheinlich (publizierte oder Labordaten); (0) = keine Daten verfügbar.

- Die Bewertung von Freisetzungsdaten erfolgte: Legende: $(+)=$ verbesserte Freisetzung in Kombination (Synergismus); (0) = keine Daten verfügbar. Die Kombination mit Gentamicin (Clindamycin) führt in der Regel zu einer besseren Freisetzung (synergistischer Effekt).

- Fosfomycin ist ein Epoxidantibiotikum und in unterschiedlichen Salzformen erhältlich. Es gilt zu beachten, dass Fosfomycin-Trometanol besser resorbierbar ist und daher den anderen verfügbaren Salzformen vorgezogen werden sollte.

- Liposomales Amphotericin B wird in niedrigen Dosierungen gut freigesetzt und sollte anderen Formen vorgezogen werden.

- Die in - Tab. 2 vorgestellten Empfehlungen sowie die zusätzlichen Erkenntnisse sind als "pocketguide“ erhältlich bzw. können von der PROIMPLANT-Foundation (Website www.pro-implant-foundation.org) elektronisch heruntergeladen werden.

Die im Konsensusmeeting 2013 vorgestellten Möglichkeiten manueller Zumischungen von Antiinfektiva zu PMMA der Arbeitsgruppe 9 in $\bullet$ Tab. 1 vorgestellten Empfehlungen basieren auf Literaturdaten und geben Hinweise darauf, welche Wirkstoffe mit PMMA kombinierbar sind. Ausdrücklich stellen diese Angaben aber keine Empfehlungen dar [56]. 


\section{Zumischempfehlungen bei} Antibiotikaüberempfindlichkeit

Bei Überempfindlichkeiten gegenüber Antibiotika gibt es einige wenige Regeln, die berücksichtigt werden müssen. Reagiert ein Patient gegen ein bestimmtes Antibiotikum allergisch, gilt die Regel, dass kein weiteres Antibiotikum derselben Klasse eingesetzt werden soll. Weiterhin ist auf Kreuzallergien zu achten, also auf eine Empfindlichkeit gegen Antibiotika anderer Klassen. Klassisches Beispiel ist die Kreuzallergie von Penicillinen und Cephalosporinen wegen der ähnlichen molekularen Grundstruktur. Sie ist mit allerdings maximal $10 \%$ niedriger als oft angenommen. Für die Beurteilung einer möglichen Antibiotikaunverträglichkeit ist auch die Erkenntnis wichtig, dass die Ergebnisse einer Epikutantestung wegen unterschiedlicher Immunreaktionen nicht unbedingt auf die Situation im Knochen und in der Gelenkhöhe $\mathrm{zu}$ übertragen sind. Grundsätzlich sind bei nachgewiesen Überempfindlichkeiten gegenüber dem im Zement befindlichen Antibiotikum eine ganze Reihe von Wirkstoffalternativen denkbar. Von daher muss nicht unbedingt auf PMMA-Zement verzichtet werden. Als einfachste Lösung kann natürlich auf einen antibiotikafreien $\mathrm{Ze}$ ment zurückgegriffen werden. Ist aber zusätzlich eine lokale Antibiose erforderlich, können die in • Tab. 3 aufgeführten Antibiotikaalternativen eingesetzt werden. Bei einer Aminoglykosidallergie kann gut auf ein Lincosamid-Antibiotikum, insbesondere auf Clindamycin zurückgegriffen werden, falls Staphylokokken Ursache für den Infekt sind. Sind sowohl Aminoglykoside als auch Lincosamide betroffen, können Colistin, Daptomycin, Linezolid und Cefuroxim (auch in Kombination) als alternative Optionen betrachtet werden. Bei Glykopeptidallergien im Fall Methicillinresistenter Staphylokokken bieten sich Carbapeneme, Linezolid, Daptomycin und Tigercylin als Alternative an.

Festzuhalten ist, dass eine Allergie gegen bestimmte Antibiotika durchaus die Verwendung von PMMA-Zement erlaubt, indem alternative Antibiotika im Zement zum Einsatz kommen.

\section{Fazit für die Praxis}

- Die systemische Antibiotikagabe bei etablierten Knochen- oder Gelenkinfektionen gilt als Therapie. Die Applikation lokaler Antibiotika ist eine adjuvante Maßnahme, die der Absicherung der Chirurgie und des Therapieerfolgs dient.

- Zu den klinisch am häufigsten eingesetzten Trägersystemen für lokale Antibiotika gehören autologes, allogenes oder synthetisches Knochenersatzmaterial, Kollagenschwämme oder Knochenzement.

- Die Freisetzung aus Knochenzement folgt den Gesetzen der Diffusion und ist signifikant abhängig von der Zementzusammensetzung und der Oberfläche der Zemente. Beim Zumischen von Antibiotika, Antiseptika, Antimykotika und Zytostatika ist stets auf antagonistische bzw. synergistische Einflüsse zu achten.

- Als Basis für eine Zumischung keine antibiotikafreien Zemente verwenden. Eine bessere Elution erreicht man durch einen bereits mit Antibiotika vorgemischten PMMA-Zement. In handelsüblichen Knochenzementen findet man derzeit lediglich 6 unterschiedliche Antibiotika (einzeln oder in Kombination).

\section{Korrespondenzadresse}

\section{Prof. Dr. K.-D. Kühn}

Universitätsklinik für Orthopädie und Orthopädische Chirurgie, Medizinische Universität Graz

Auenbruggerplatz 5, $8036 \mathrm{Graz}$, Österreich Klaus.kuehn@medunigraz.at

Open access funding provided by Medical University of Graz.

\section{Einhaltung ethischer Richtlinien}

Interessenkonflikt. K.-D. Kühn, N. Renz und A. Trampuz geben an, dass kein Interessenkonflikt besteht.

Dieser Beitrag beinhaltet keine von den Autoren durchgeführten Studien an Menschen oder Tieren.

Open Access. Dieser Artikel wird unter der Creative Commons Namensnennung 4.0 International Lizenz (http://creativecommons.org/licenses/by/4.0/deed. de) veröffentlicht, welche die Nutzung, Vervielfältigung, Bearbeitung, Verbreitung und Wiedergabe in jeglichem Medium und Format erlaubt, sofern Sie den/die ursprünglichen Autor(en) und die Quelle ordnungsgemäßnennen, einen Link zur Creative Commons Lizenz beifügen und angeben, ob Änderungen vorgenommen wurden.

\section{Literatur}

1. Akiyama T, Hiroshi M, Yutaka $Y$, Masatsugu T, Yoshiki A, Iwao N, Motoki S, Masaaki M (2013) Silver oxide-containing hydroxyapatite coating has in vivo antibacterial activity in the rat tibia. J Orthop Res 31(8):1195-1200(10.1002/jor.22357)

2. AmerstorferF,FischerauerS, SadoghiP, Schwantzer G, Kühn KD, Leithner A, Glehr M (2016) Superficial vancomycin coating of bone cement in orthopedic revision surgery: a safe technique to enhance loca antibiotic concentrations. J Arthroplasty. doi:10. 1016/j.arth.2016.11.042

3. Bennett-Guerrero E, Pappas TN, Koltun WA, Fleshman JW, Lin M, Garg J, Mark DB, Marcet JE, Remzi FH, George VV, Newland K, Corey GR, SWIPE 2 Trial Group (2010) Gentamicincollagen sponge for infection prophylaxis in colorectal surgery. NEngl J Med 363(11):1038-1049

4. Bösebeck H, Büchner H (2010) Struktur, Wirkmechanismen und Einsatzgebiete neuer Knochenersatzsubstanzen und Knochenregenerationsmaterialien. In: Peters KM, König DP (Hrsg) Fortbildung Osteologie. Springer, Berlin Heidelberg, S 109-115

5. Bryson DJ, Morris DL, Shivji FS, Rollins KR, Snape S, Ollivere BJ (2016) Antibiotic prophylaxis in orthopaedic surgery: difficult decisions in an era of evolving antibiotic resistance. Bone Joint J 98B(8):1014-1019

6. Breusch SJ, Kühn KD (2003) Bone cements based on polymethylmethacrylate. Orthopaede 32(1):41-50

7. Buchholz HW, Engelbrecht $H$ (1970) Depot effects of various antibiotics mixed with Palacos resins. Chirurg 41:511-515

8. Chang YH, Tai CL, Hsu HY, Hsieh PH, Lee MS, Ueng SWN (2014) Liquid antibiotics in bone cement. Bone Joint Res 3(8):246-251. doi:10.1302/20463758.38.2000305

9. Coraça-Huber DC, Wurm A, Fille M, Hausdorfer J, Nogler M, Kühn KD (2014a) Effect of freezing on the release rate of gentamicin palmitate and gentamicin sulfate from bone tissue. J Orthop Res 32(6):842-847. doi:10.1002/jor.22602

10. Coraça-Huber DC, Putzer D, Fille M, Hausdorfer J, Nogler M, Kühn KD (2014b) Gentamicin palmitate as a new antibiotic formulation for mixing with bone tissue and local release. Cell Tissue Bank 15(1):139-144

11. Coraça-Huber DC, Ammann CG, Nogler M, Wurm A, Fille M, Frommelt L, Kühn KD, Fölsch C (2015a) Lyophilized allogeneic bone tissue as an antibiotic carrier. Cell Tissue Bank. doi:10.1007/s10561-0169582-5

12. Coraça-Huber DC, Wurm A, Fille M, Hausdorfer J, Nogler M, Vogt S, Kühn KD (2015b) Antibiotic-loaded calcium carbonate/calcium sulfate granules as co-adjuvant for bone grafting. J Mater Sci Mater Med 26(1):5344

13. Cottagnoud P, Cottagnoud M, Tauber MG (2003) Vancomycin acts synergistically with gentamicin against penicillin-resistant pneumococci by increasing the intracellular penetration of gentamicin. Antimicrob Agents Chemother 47(1):144-147

14. de Boer HH (1988) The history of bone grafts. Clin Orthop Relat Res 226:292-298 
15. Drago L, Boot W, Dimas K, Malizos K, Hänsch GM, Stuyck J, Gawlitta D, Romanò CL (2014) Does implant coating with antibacterial-loaded hydrogel reduce bacterial colonization and biofilm formation in vitro? Clin Orthop Relat Res 472(11):3311-3323

16. Engesaeter LB, LieSA, Espehaug B, Furnes O, Vollset SE, Havelin LI (2003) Antibiotic prophylaxis in total hip arthroplasty: effects of antibiotic prophylaxis systemically and in bone cement on the revision rate of 22,170 primary hip replacements followed $0-14$ years in the Norwegian Arthroplasty Register. Acta Orthop Scand 74(6):644-651

17. Fink B, Vogt S, Reinsch M, Büchner H (2011) Sufficient release of antibiotic by a spacer 6 weeks after implantation in two-stage revision of infected hip prostheses. Clin Orthop Relat Res 469(11):3141-3147

18. Fleischman AN, Austin MS (2017) Local intrawound administration of powdered antibiotics in orthopaedic surgery. J Bone Joint Infect 2(1):23-28. doi:10.7150/jbji.16649

19. Fleiter N, Walter G, Bösebeck H, Vogt S, Büchner H HirschbergerW, Hoffmann R(2014)Clinical use and safety of a novel gentamicin-releasing resorbable bone graft substitute in the treatment of osteomyelitis/osteitis. Bone Joint Res 3(7):223-229. doi:10.1302/2046-3758.37.2000301

20. Frommelt $L$ (2004a) Prinzipien der Antibiotikabehandlung bei periprothetischen Infektionen. Orthopäde 33(7):822-828

21. Frommelt $L$ (2004b) Lokale Antibiotikatherapie. In: Schnettler R, Steinau HU (Hrsg) Septische Knochenchirurgie. Thieme, Stuttgart New York, S 82-89. ISBN 978-3131169815

22. Frommelt L, Kühn KD (2005) Antibiotic-loaded cement. In: Breusch SJ, Malchau M (Hrsg) The wellcemented total hip arthroplasty. Springer, Berlin Heidelberg, S86-92

23. Frommelt $L$ (2007) Antibiotic choices in bone surgery - local therapy using antibiotic-loaded bone cement. In: Walenkamp GHIM (Hrsg) Local antibiotics in arthroplasty. State of the art from an interdisciplinary view. Thieme, Stuttgart New York, S59-64. ISBN 978-1588906076

24. Gallo J, Holinka M, Moucha CS (2014) Antibacteria surface treatment for orthopaedic implants. Int J Mol Sci 15(8):13849-13880. doi:10.3390/ ijms150813849

25. Gollwitzer $\mathrm{H}$, Ibrahim K, Meyer H, Mittelmeier W, Busch R, Stemberger A (2003) Antibacterial poly (D,L-lactic acid) coating of medical implants using a biodegradable drug delivery technology. J Antimicrob Chemother 51(3):585-591

26. Hanssen AD (2004) Prophylactic use of antibiotic bone cement: an emerging standard-in opposition. J Arthrop 19(4Suppl1):73-77. doi:10.1016/j. arth.2004.04.006

27. Hanssen AD, Spangehl MJ (2004) Treatment of the infected hip replacement. Clin Orthop Relat Res 420:63-71

28. Hickson CJ, Metcalfe D, Elgohari S, Oswald T, Masters JP, Rymaszewska M, Reed MR, Sprowson AP (2015) Prophylactic antibiotics in elective hip and knee arthroplasty - an analysis of organisms reported to cause infections and national survey of clinical practice. Bone Joint Res 4(11):181-189. doi:10.1302/2046-3758.411.2000432

29. Hsieh PH, Huang KC, Tai CL (2009) Liquid gentamicin in bone cement spacers: in vivo antibiotic release and systemic safety in two-stage revision of infected hip arthroplasty. J Trauma 66(3):804-808
30. ISO 5833:2002: Implants for Surgery-Acrylic Resin Cements. Orthopaedic Application, Beuth Verlag

31. Jämsen E, Stogiannidis I, Malmivaara A, Pajamäki J, Puolakka TT, Konttinen YT (2009) Outcome of prosthesis exchange for infected knee arthroplasty: the effect of treatment approach. Acta Orthop 80(1):67-77

32. Kabata T, Maeda T, Kajino Y, Hasegawa K, Inoue D, Yamamoto T, Takagi T, Ohmori T, Tsuchiya H (2015) lodine-supported hip implants: short term clinica results. Biomed Res Int. doi:10.1155/2015/368124

33. Khan NR, Thompson CJ, DeCuypere M, Angotti JM, Kalobwe E, Muhlbauer MS, Camillo FX, Klimo P Jr. (2014) A meta-analysis of spinal surgical site infection and vancomycin powder. J Neurosurg Spine 21(6):974-983

34. Klemm K (1979) Gentamicin-PMMA-beads in treating bone and soft tissue infections (author's transl). Zentralbl Chir 104(14):934-942

35. Klemm KW (1993) Antibiotic bead chains. Clin Orthop 295:63-76

36. Kühn KD (2001) Knochenzemente für die Endoprothetik. Springer, Berlin Heidelberg. ISBN 9783540411826

37. Kühn KD (2014a) PMMA Cements. Are we aware what we are using? Springer, Berlin Heidelberg doi:10.1007/978-3-642-41536-4.ISBN 978-3642415357

38. Kühn KD (2014b) Antimicrobial ImplantCoating. In: Scholz M (Hrsg) Biofunctional surface engineering. Pan Stanford, Singapore, S 121-189. ISBN 9789814411608

39. Kühn KD, Höntzsch D (2015) Augmentation with PMMA cement. Unfallchirurg 118(9):737-748. doi:10.1007/s00113-015-0059-y

40. Kühn KD, Lieb E, Berberich C (2016) PMMA bone cement: what is the role of local antibiotics? Maîtrise Orthop 243:12-18

41. Lautenschlager EP, Jacobs JJ, Marshall GW, Meyer PR Jr (1976a) Mechanical properties of bone cements containing large doses of antibiotic powders. JBiomed Mater Res 10(6):929-938

42. Lautenschlager EP, Marshall GW, Marks KE, Schwartz J, Nelson CL (1976b) Mechanical strength of acrylic bone cements impregnated with antibiotics. JBiomed Mater Res 10(6):837-845

43. Mcnally $M$, Ferguson J, Kendall J, Dudareva M, Scarborough M, Stubbs D (2015) A comparative study of three bioabsorbable antibiotic carriers in chronic osteomyelitis: 313 patients with minimum one-year follow-up. Bone Joint J 97-B(supp 16):21

44. Mackey D, Varlet A, Debeaumont D (1982) Antibiotic loaded plaster of Paris pellets: an in vitro study of a possible method of local antibiotic therapy in bone infection. Clin Orthop 167:263-268

45. Molinari RW, Khera OA, Molinari WJ 3rd (2012) Prophylactic intraoperative powdered vancomycin and postoperative deep spinal wound infection: 1,512 consecutive surgical cases over a 6-year period. Eur Spine J 21(Suppl 4):S476-S482

46. Mulazimoglu L, Drenning SD, Muder RR (1996) Vancomycin-gentamicin synergism revisited: effect of gentamicin susceptibility of methicillinresistant Staphylococcus aureus. Antimicrob Agents Chemother 40(6):1534-1535

47. Marczak D, Synder M, Sibiński M, Okoń T, Kowalczewski J (2015) The use of calciumcarbonate beads containing gentamicin in the second stage septic revision of total knee arthroplasty reduces reinfection rate. Knee 23(2):322-326. doi:10.1016/ j.knee.2015.12.001

48. Matl FD, Obermeier A, Repmann S, Friess W, Stemberger A, Kühn KD (2008) New anti-infective coatings of medical implants. Antimicrob Agents Chemother 52(6):1957-1963

49. Murphy EP, Curtin M, Shafqat A, Byrne $F$, Jadaan $M$, Rahall E (2017) A review of the application of vancomycin powder to posterior spinal fusion wounds with a focus on side effects and infection. A prospective study. Eur J Orthop Surg Traumatol 27(2):187-191. doi:10.1007/s00590-016-1878-4

50. Obermeier A, Matl FD, Schwabe J, Zimmermann A, Kühn KD, Lakemeier $S$, von Eisenhart-Rothe R, Stemberger A, Burgkart R (2012) Novel fatty acid gentamicin salts as slow-release drug carrier systems for anti-infective protection of vascular biomaterials. J Mater Sci Mater Med 23(7):1675-1683

51. Obermeier A, Schneider J, Föhr P, Wehner S, Kühn KD, Stemberger A, Schieker M, Burgkart R (2015) In vitro evaluation of novel antimicrobial coatings for surgical sutures using octenidine. BMC Microbiol. doi:10.1186/s12866-015-0523-4

52. Ochsner PE, Borens O, Bodler PM, Broger I, Eich G, Maurer T, Nötzli H, Trampuz A, Uchay I, Vogt M, ZimmerliW (2013) Infektion des Bewegungsapparates. Herausgegeben durch die Expertengruppe Infektionen des Bewegungsapparates der Schweiz. Gesellschaft für Orthopädie und Traumatologie (swiss orthopaedics) und der Schweiz. Gesellschaft für Infektiologie SGInf, 1. Aufl. Eigenverlag swiss orthopaedics, Grandvaux

53. Ostermann PA, Seligson D, Henry SL (1995) Local antibiotic therapy for severe open fractures. A review of 1085 consecutive cases. JBone Joint Surg Am 77(1):93-97

54. Parikh SN (2002) Bone graft substitutes in modern orthopedics. Orthopedics 25(11):1301-1309

55. Parvizi J, Saleh KJ, Ragland PS, Pour AE, Mont MA (2008) Efficacy of antibiotic-impregnated cement in total hip replacement. Acta Orthop 79(3):335-342

56. Parvizi J, Gehrke T (2013) Proceedings of the international consensus meeting on periprosthetic joint infection. Data Trace Publishing Company, Brooklandville. ISBN 978-1574001471

57. Pahys JM, Pahys JR, Cho SK, Kang MM, Zebala LP, Hawasli AH, Sweet FA, Lee DH, Riew KD (2013) Methods to decrease postoperative infections following posterior cervical spine surgery. J Bone Joint Surg Am 95(6):549-554. doi:10.2106/JBJS.K. 00756

58. Patzakis MJ, Mazur K, Wilkins J, Sherman R, Holtom P (1993) Septopal beads and autogenous bone grafting for bone defects in patients with chronic osteomyelitis. Clin Orthop 295:112-118

59. Peel TN, Cheng AC, Buising KL, Choong PF (2012) Microbiological aetiology, epidemiology, and clinical profile for prosthetic joint infections: are current antibiotic prophylaxis guidelines effective? Antimicrob Agents Chemother 56(5):2386-2391

60. Renz N, Perka C, Trampuz A (2016) Management periprothetischer Infektionen des Kniegelenks. Orthopäde45(1):65-71.doi:10.1007/s00132-0153217-6

61. Renz N, Trampuz A (2017) Pro-Implant Foundation Pocket Guide, Version 5 23. www.pro-implantfoundation.org

62. Romanò CL, Scarponi S, Gallazzi E, Romanò D, Drago L (2015) Antibacterial coating of implants in orthopaedics and trauma: a classification proposal in an evolving panorama.J Orthop Surg Res. doi:10. 1186/s13018-015-0294-5

63. Schnürer SM, Gopp U, Kühn KD, Breusch SJ (2003) Knochenersatzwerkstoffe. Orthopäde 32:2-10

64. Sohail MR, Uslan DZ, Khan AH, Friedman PA, Hayes DL, Wilson WR, Steckelberg JM, Stoner S, 
Baddour LM (2007) Management and outcome of permanent pacemaker and implantable cardioverter-defibrillator infections. J Am Coll Cardiol 49(18):1851-1859

65. Schmidmaier G, Lucke M, Wildemann B, Haas NP, Raschke M (2006) Prophylaxis and treatment of implant-related infections by antibiotic-coated implants: a review. Injury 37(Suppl2):105-112. doi:10.1016/j.injury.2006.04.016

66. Shirai T, Tsuchiya H, Nishida H, Yamamoto N, Watanabe K, Nakase J, Terauchi R, Arai Y, Fujiwara H, Kubo T (2014) Antimicrobia megaprostheses supported with iodine.JBiomater Appl 29(4):617-623

67. Thallinger B, Prasetyo EN, Nyanhongo GS, Guebitz GM (2013) Antimicrobial enzymes: an emerging strategy to fight microbes and microbial biofilms. Biotechnol J 8(1):97-109

68. Vogt S, Kühn KD, Gopp U, Schnabelrauch M (2005) Resorbable antibiotic coatings for bone substitutes and implantable devices. Materwiss Werksttech 36:814-819

69. Wahlig H (1987) Über die Freisetzungskinetik von Antibiotika aus Knochenzementen - Ergebnisse vergleichender Untersuchungen in vitro und in vivo. In: Willert HG, Buchhorn G (Hrsg) principles, design and saftety of joint implantr. Hogrefe \& Huber, Göttingen, S 221-226. ISBN 9780889370906

70. Winkler H, Stoiber A, Kaudela K, Winter F, Menschik $F$ (2008) One stage uncemented revision of infected total hip replacement using cancellous allograft bone impregnated with antibiotics. J Bone Joint Surg Br 90(12):1580-1584. doi:10. 1302/0301-620X.90B12.20742

\section{K.C. Chung (Ed.) \\ Clinical Management of the Rheumatoid Hand, Wrist, and Elbow}

\section{Springer International Publishing}

2016, 1. Auflage, 330 S., 260 Abb., (ISBN: 978-3-319-26658-9), Hardcover 160,49 EUR, eBook (978-3-319-266602) 118,99 EUR

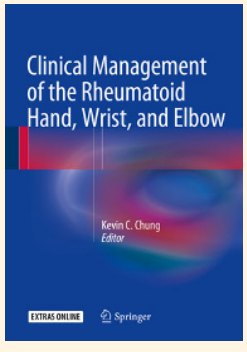

Die Erstausgabe

"Clinical Management of the Rheumatoid Hand, Wrist and Elbow" ist ein über 300-seitiges Kompendium zur operativen Therapie der Hand, des

Handgelenkes und des Ellenbogens bei rheumatoider Arthritis. Das Buch ist in vier große Bereiche gegliedert (Hintergrundwissen, rheumatisches Handgelenk, rheumatische Hand, rheumatischer Ellenbogen). Im ersten Kapitel werden Hintergründe zur rheumatoiden Arthritis dargestellt. Dies inkludiert die Mechanismen der neuesten Medikamente, mögliche Pathomechanismen der rheumatoiden Arthritis und wichtige Felder wie z.B. die prä- und postoperative Medikamententherapie beim chirurgischen Eingriff. Ein eigenes Kapitel für die Indikation und den Zeitpunkt des chirurgischen Eingriffs beim Patienten mit rheumatoider Arthritis wird ebenso suffizient dargestellt, wie verschiedene Untersuchungsscores. Der zweite Bereich befasst sich mit dem rheumatischen Handgelenk. Hier werden zunächst verschiedene biomechanische Erklärungsmodelle und Konzepte zum rheumatischen Handgelenk aufgeführt. Danach erfolgt eine detaillierte Beschreibung der verschiedenen Handgelenksregionen inklusive des distalen Radioulnargelenkes. Der Bereich des rheumatischen Handgelenkes wird abgerundet mit einer hervorragenden Falldiskussion, bei der verschiedene Patienten kurz und prägnant dargestellt und dann, nach Abarbeiten der Diagnostik, die entsprechenden Therapieoptionen aufgeführt werden. Hierdurch ist eine Wissensüberprüfung des Lesers möglich. Im dritten Teil des Buches wird die rheumatoide Handdeformität dargestellt. Hier ebenfalls Beginn mit biomechanischen Überlegun- gen zur Entstehung der Deformitäten und Indikationsstellung. In Folge werden neben den Gelenkersatzmöglichkeiten auch die wichtigen Sehneneingriffe vorgestellt. Auch dieser Bereich wird wiederum durch eine fallbasierte Darstellung von verschiedenen Patienten abgerundet. Als letzter Bereich wird, etwas kürzer als die vorhergehenden Anteile, der rheumatische Ellenbogen und die Therapiemöglichkeiten dargestellt.

Die Indikation zu hand- bzw. ellenbogenchirurgischen Eingriffen bei Patienten mit rheumatoider Arthritis ist rückläufig. Nichtsdestotrotz können weiterhin viele Patienten von der operativen Therapie profitieren. Hierzu stellt dieses Buch einen hervorragenden Leitfaden dar. Aufgrund der sorgfältig ausgearbeiteten Kapitel, der sinnvollen Gliederung und der ansprechenden Abbildungen kann dieses Buch jedem Chirurgen, der eine operative Therapie bei rheumatoiden Patienten durchführt, nur wärmstens empfohlen werden. Auch internistische Rheumatologen können aufgrund der guten Darstellung der operativen Techniken von diesem Buch durchaus profitieren.

F. Unglaub (Bad Rappenau) 